\title{
Human T- Lymphotropic Virus Type 1 in Blood Donors from Babol County Blood Transfusion Center: A Pilot Study From Northern Iran
}

\author{
Yousef Yahyapour, ${ }^{1}$ Kazem Aghajanipour, ${ }^{2}$ Seyed Mostafa Mir, ${ }^{3}$ Aynaz Khademian, ${ }^{4}$ and Farzin \\ Sadeghi ${ }^{5,}$, \\ ${ }^{1}$ Infectious Diseases and Tropical Medicine Research Center, Babol University of Medical Sciences, Babol, Iran \\ ${ }^{2}$ Babol County Blood Transfusion Center, Babol, Iran \\ ${ }^{3}$ Department of Clinical Biochemistry, Faculty of Medicine, Babol University of Medical Sciences, Babol, Iran \\ ${ }^{4}$ Department of Microbiology, Faculty of Medicine, Babol University of Medical Sciences, Babol, Iran \\ ${ }^{5}$ Cellular and Molecular Biology Research Center, Health Research Institute, Babol University of Medical Sciences, Babol, Iran \\ "Corresponding author: Farzin Sadeghi, Cellular and Molecular Biology Research Center, Health Research Institute, Babol University of Medical Sciences, Babol, Iran. Tel/Fax: \\ +98-1132196476, E-mail: sadeghifarzin6@gmail.com
}

Received 2017 March 03; Revised 2017 May 21; Accepted 2017 June 24.

\begin{abstract}
Background: Human T-lymphotropic virus type 1(HTLV-1) infection is a major health problem that effects a variety of endemic regions, including the Northeast of Iran. There is a lack of information about HTLV-1 prevalence among blood donors from Mazandaran province, Northern Iran.

Objectives: The aim of the present study was to investigate the prevalence of HTLV-1 infection among blood donors in Babol County, the most populated county in Mazandaran province with screening and confirmatory assays.

Methods: The present cross-sectional study was conducted on 503 blood donors. Serum samples from each blood donor were screened for HTLV-1 specific antibodies using the Enzyme-Linked Immunosorbent Assay (ELISA). Samples that were repeatedly reactive for HTLV-1 specific antibodies on serological screening were additionally confirmed by real time polymerase chain reaction (PCR) of HTLV-1 proviral DNA in Peripheral Blood Mononuclear Cells (PBMCs).

Results: Among 503 samples tested by serological enzyme linked immunosorbent assay (ELISA), 13 samples (2.6\%) were repeatedly reactive (i.e. on at least 2 of 3 occasions). All repeatedly reactive samples were examined for the presence of the HTLV-1 proviral DNA in PBMCs by real time PCR confirmatory test, of which 1 sample was positive, resulting in HTLV-1 prevalence of $0.2 \%$.

Conclusions: The present investigation contributes with new epidemiologic data reporting low prevalence rate for $H T L V-1$ among blood donors in Babol county of the Mazandaran province. Despite the low prevalence rate, the practice of screening of donated bloods in blood transfusion centers of Mazandaran province should be considered to decrease the risk of virus transmission in this region.
\end{abstract}

Keywords: Blood Donor, Real Time Polymerase Chain Reaction, Human T-lymphotropic virus 1

\section{Background}

Human T-lymphotropic virus type 1 (HTLV-1) is a worldwide health problem and is the etiological agent for aggressive T-cell malignancy, known as Adult T-cell Leukemia/Lymphoma (ATL) as well as HTLV-1-associated myelopathy/tropical spastic paraparesis (HAM/TSP) (1). It has been estimated that 5 to 10 million people are infected with HTLV-1 worldwide (2). Human T-lymphotropic virus type 1 infection has been recognized throughout the world, yet is found predominantly in sub-Saharan Africa, south-west Japan, Caribbean basin, parts of South America, the Melanesian Islands, and the Middle East (3). From an epidemiological standpoint, there are 3 major routes for HTLV-1 transmission: mother-to-child transmission through breast feeding, sexual transmission, and parenteral transmission through transfusion of contami- nated blood (4). Blood transfusion, although amenable to prevention efforts, continue to be an efficient transmitter of $H T L V-1$ infection. Transmission rate of $H T L V-1$ followed by transfusion of contaminated cellular blood components is estimated to be between $27 \%$ and $63 \%$ (5).

In the Middle East region, Northeast of Iran (Razavi Khorasan province) is known to be endemic for HTLV-1. Seroprevalence of $H T L V$ - 1 infection among blood donors in Mashhad, the capital of Razavi Khorasan province and the main pilgrimage city for Shiites, was estimated as approximately $2 \%$ (6). Screening of blood donors for HTLV-1 antibodies is mandatory in 7 provinces of Iran, including Northern Khorasan, southern Khorasan, Ardabil, Alborz, Gilan, Western Azerbaijan, and Razavi Khorasan, and is not obligatory in other provinces of Iran (7). Although, HTLV-1 seroprevalence in blood donors has a much lower rate in 
other parts of Iran, based on a recently published systematic review there is no information about $H T L V$-1 prevalence among blood donors from Mazandaran province, which is one of the most densely populated provinces in Northern Iran and is thought to have a great number of pilgrims to Mashhad every year (8).

\section{Objectives}

The aim of the present study was to investigate the prevalence of HTLV-1 infection among blood donors in Babol county, the most populated county of the Mazandaran province, and to evaluate seropositive samples with real time PCR confirmatory assay to rule out potential falsepositive results.

\section{Methods}

\subsection{Ethics statement}

This study was approved by the ethical committee of Babol University of Medical Sciences, and written informed consent was obtained from all subjects (University Ethics Committee code: MUBABOL.REC.1395.6).

\subsection{Study Population}

The present cross-sectional study was conducted on blood volunteers, who had been referred to blood transfusion centers of Babol county, between December 2015 and February 2016. This county is located in the province of Mazandaran, the northern province of Iran and subdivided to seven districts. According to a Census in 2012, Babol county has registered a population of 495472 inhabitants and the rural population constitutes $48 \%$ of the total inhabitants. All individuals fulfilling the following criteria were included in the current study: 1) age of 18 to 65 years, 2) lack of risky behavior for blood born viral infections, including unsafe sexual practices and use of illegal drugs injection. The criteria for exclusion of donors were 1 ) age of $<$ 18 years or $>65$ years 2 ) history of unsafe sexual practices and use of illegal drug injection. A total of 503 blood samples were collected from blood donors during the aforementioned period. Donor demographic characteristics including age, gender, urban/rural residence status, marital status, level of educational attainment, and district of residence were recorded at the time of donation.

\subsection{Sample Collection and Processing}

About 6-mL of peripheral blood was collected from each donor in sterile vacutainer tubes and then serum was separated by centrifugation and stored at $-80^{\circ} \mathrm{C}$ until use. Peripheral blood mononuclear cells (PBMCs) of the blood samples were isolated by a standard procedure using Ficoll (Lympholyte H, Cedarlane, Hornby, Canada) density gradient centrifugation (9), and the cells were counted and resuspended in $200 \mu \mathrm{L}$ of RNALater solution (Behnogen, Tehran, Iran) at $-20^{\circ} \mathrm{C}$ until use.

\subsection{Serological Screening}

Serum samples from each blood donor were screened for HTLV-1-specific antibodies using the Enzyme-Linked Immunosorbent Assay (ELISA) method (Dia. Pro Diagnostic Bioprobes, Milan, Italy). This assay uses HTLV-1specific antigen-coated microplates (gp46-I and p21-I) in solid phase and antibody sandwich technique. Assay interpretation was done using a conventional colorimetric readout. Samples with ELISA optical density to cut-off ratios below 0.9 were considered negative, between 0.9 and 1.1 indeterminate, and higher than 1.1 were considered positive.

\subsection{DNA Isolation}

Genomic DNA was extracted from MT-2 HTLV-1 infected cell line (as a positive control) and PBMC specimens using YTA Genomic DNA Extraction Mini Kit (Yekta Tajhiz Azma, Tehran, Iran), according to the manufacturer's instructions. The quality and quantity of purified DNA was determined using a NanoDrop spectrophotometer (Thermo Scientific, Wilmington, USA). To monitor the efficacy of DNA isolation, for each extraction, DNA integrity was evaluated using human RNase P gene (RPP30) amplification, based on a previously described procedure (10). Sterile microcentrifuge tubes containing only reaction mixtures were processed simultaneously with PBMC specimens as a DNA isolation negative control. The HTLV-1 infected human lymphoblastoid cell line MT-2 was obtained from the Pasteur institute (Iran).

\subsection{Real Time Polymerase Chain Reaction Confirmatory Assay}

Samples that were repeatedly reactive for HTLV-1 specific antibodies on serological screening were additionally confirmed by real time PCR of HTLV-1 proviral DNA in PBMCs. Real-time PCR was performed using an ABI 7300 Real-Time PCR System (Applied Biosystems, Branchburg, NJ,USA) and the following genomic regions were amplified by the primer sets and TaqMan probe, $115 \mathrm{bp}$ of the most conserved region of tax gene of HTLV-1 and 65 bp of human RNase P gene (RPP30) for internal calibration (10, 11). All primers were synthesized by Metabion International AG (Martinsried, Germany). Each reaction consisted of $100 \mathrm{ng}$ of extracted DNA, 12.5 $\mu$ L YTA 2X Multiplex Real-Time PCR Smart mix (Yekta TajhizAzma, Iran), $0.3 \mu \mathrm{M}$ of final concentration for each primer, and $0.2 \mu \mathrm{M}$ of final concentration 
of each probe in a $25-\mu \mathrm{L}$ total reaction volume. Real-time PCR was conducted in two steps with the following thermal setting: $95^{\circ} \mathrm{C}$ for 5 minutes $\left(1\right.$ cycle), $95^{\circ} \mathrm{C}$ for $30 \mathrm{sec}-$ onds, and $60^{\circ} \mathrm{C}$ for 45 seconds ( 40 cycles). Each real-time PCR run included reaction mixtures without DNA template as a negative control and DNA extracted from MT-2 HTLV-1infected human lymphoblastoid cell line as a positive control.

\section{Results}

A total of 503 blood donors that ranged in age from 19 to 61 years were included in the final sample population. Four hundred and eighty-three of them were males (96.0\%) and 20 (4.0\%) were females. The mean age of males was $40.8 \pm 9.8$ and for females this was $41.2 \pm 11.4$. Demographic characteristics of donors are shown in Table 1. All 503 serum samples were screened for anti-HTLV-1 antibodies. Among total samples tested by serological ELISA, 13 samples (2.6\%) were repeatedly reactive (i.e. on at least 2 of 3 occasions). All repeatedly reactive samples were examined for the presence of the HTLV-1 proviral DNA in PBMCs by real time PCR confirmatory test, of which one sample was positive, resulting in $H T L V-1$ prevalence of $0.2 \%$.

The only HTLV-1 positive blood donor was a 34-yearold married male, who was born in the central district of Babol. Based on his past medical history, he was admitted to one of the Tehran hospitals in 2004 for pulmonary infection and received 2 units of whole blood. The aforementioned blood donor was notified and in accordance with the Iranian blood transfusion organization guidelines, he was permanently deferred from blood donation. In addition, the donor blood components were discarded, and he remains ineligible for future blood donation.

\section{Discussion}

In the current investigation, the authors report the prevalence of HTLV-1 infection among blood donors in Babol County, the most populated county of the Mazandaran province. As far as the authors are concerned, the present study was the largest to date among blood donors in Mazandaran province, which utilized real time PCR confirmatory assay to rule out potential false-positive results for $H T L V-1$ infection. The results of this study showed that HTLV-1 prevalence among blood donors of Babol was $0.2 \%$. This finding showed that the rate of $H T L V-1$ infection in blood donors of Babol is lower than infection rate in endemic areas, such as Mashhad, Neyshbour, Sabzevar, and other non-endemic provinces, such as West Azarbaijan and Charmahal-Bakhtiari $(6,12-15)$. However, the current results were almost similar to the HTLV-1 prevalence in blood
Table 1. Demographic Characteristics of Studied Blood Donors in Babol County

\begin{tabular}{|c|c|c|}
\hline Characteristics & Number & Percent \\
\hline \multicolumn{3}{|l|}{ Gender } \\
\hline Male & 483 & 96.0 \\
\hline Female & 20 & 4.0 \\
\hline \multicolumn{3}{|l|}{ Age, y } \\
\hline $19-29$ & 66 & 13.1 \\
\hline $30-39$ & 162 & 32.2 \\
\hline $40-49$ & 164 & 32.6 \\
\hline$\geq 50$ & 111 & 22.1 \\
\hline \multicolumn{3}{|l|}{ Residence status } \\
\hline Urban & 346 & 68.8 \\
\hline Rural & 157 & 31.2 \\
\hline \multicolumn{3}{|l|}{ Marital status } \\
\hline Single & 47 & 9.3 \\
\hline Married & 456 & 90.7 \\
\hline \multicolumn{3}{|l|}{ Education level } \\
\hline Illiterate & 7 & 1.4 \\
\hline Primary school & 208 & 41.4 \\
\hline High school diploma & 155 & 30.8 \\
\hline Academic education & 133 & 26.4 \\
\hline \multicolumn{3}{|l|}{ Babol districts } \\
\hline Central & 352 & 70.0 \\
\hline Amirkola & 29 & 5.8 \\
\hline Lalehabad & 31 & 6.2 \\
\hline Gatab & 47 & 9.3 \\
\hline Babol Kenar & 12 & 2.4 \\
\hline Western Bandpey & 12 & 2.4 \\
\hline Eastern Bandpey & 20 & 4.0 \\
\hline
\end{tabular}

donors of provinces, such as Alborz, Ilam, and Hormozgan (8). In Mazandarani blood donors, there is only one seroprevalence pilot study with limited sample size, which has reported $1.8 \%$ seroprevalence rate for $H T L V-1$ (16).

It is quite likely that results of the aforementioned pilot study in Mazandaran was prone to false-positive effects, due to a lack of confirmatory assay in sero-reactive subjects. In addition, in a recently published study on Mazandarani thalassemia patients, screening by ELISA showed 6.9\% HTLV-1 seroprevalence rate, yet after PCR confirmatory assay, only $1.4 \%$ of patients were HTLV-1 true positive (17). Considering the false positive samples, all possible strategies to diminish false positive results should be implemented, including the use of real time PCR confirma- 
tory tests. In the current investigation, all repeatedly seroreactive samples were tested for the presence of $H T L V-1$ proviral DNA by TaqMan real time PCR technology. Molecular techniques such as TaqMan real time PCR technology with high level of specificity are one of the most reliable procedures for clinical confirmation of HTLV-1 infection (11).

\subsection{Conclusion}

In conclusion, the present investigation contributes to new epidemiologic data reporting low prevalence rate for HTLV-1 among blood donors in Babol county of the Mazandaran province. Despite the low prevalence rate, the practice of screening of donated bloods in the blood transfusion centers of Mazandaran province should be expected to decline the risk of virus transmission in this region.

\section{Acknowledgments}

The authors thankfully acknowledge the directors and staff of Babol county blood transfusion center for their collaboration in sample collection, especially Maryam Nazoktabar.

\section{Footnotes}

Authors' Contribution: Yousef Yahyapour and Farzin Sadeghi developed the study concepts, experimental protocols, and prepared the manuscript. Kazem Aghajanipour, Seyed Mostafa Mir, and Aynaz Khademian carried out experimental protocols and sample collection.

Financial Disclosure: This study was financially supported by a grant from Babol University of Medical Sciences (Project code: 9339424).

Conflict of Interests: The authors declare that they had no conflicts of interest.

\section{References}

1. Plummer M, de Martel C, Vignat J, Ferlay J, Bray F, Franceschi S. Global burden of cancers attributable to infections in 2012: a synthetic analysis. Lancet Glob Health. 2016;4(9):e609-16. doi: 10.1016/S2214109X(16)30143-7. [PubMed: 27470177].

2. Gessain A, Cassar O. Epidemiological Aspects and World Distribution of HTLV-1 Infection. Front Microbiol. 2012;3:388. doi: 10.3389/fmicb.2012.00388. [PubMed: 23162541].

3. Proietti FA, Carneiro-Proietti AB, Catalan-Soares BC, Murphy EL. Global epidemiology of HTLV-I infection and associated diseases. Oncogene. 2005;24(39):6058-68. doi:10.1038/sj.onc.1208968. [PubMed:16155612].
4. Goncalves DU, Proietti FA, Ribas JG, Araujo MG, Pinheiro SR, Guedes $\mathrm{AC}$, et al. Epidemiology, treatment, and prevention of human $\mathrm{T}$ cell leukemia virus type 1-associated diseases. Clin Microbiol Rev. 2010;23(3):577-89. doi: 10.1128/CMR.00063-09. [PubMed: 20610824].

5. Chang YB, Kaidarova Z, Hindes D, Bravo M, Kiely N, Kamel H, et al. Seroprevalence and demographic determinants of human Tlymphotropic virus type 1 and 2 infections among first-time blood donors-United States, 2000-2009. JInfect Dis. 2014;209(4):523-31. doi: 10.1093/infdis/jit497. [PubMed: 24068702].

6. Rafatpanah H, Hedayati-Moghaddam MR, Fathimoghadam F, Bidkhori HR, Shamsian SK, Ahmadi S, et al. High prevalence of HTLV-I infection in Mashhad, Northeast Iran: a population-based seroepidemiology survey. J Clin Virol. 2011;52(3):172-6. doi: 10.1016/j.jcv.2011.07.004. [PubMed: 21840754].

7. Hedayati-Moghaddam MR, Tehranian F, Bayati M. Human TLymphotropic Virus Type I (HTLV-1) Infection among Iranian Blood Donors: First Case-Control Study on the Risk Factors. Viruses. 2015;7(11):5736-45. doi: 10.3390/v7112904. [PubMed: 26556363].

8. Hedayati-Moghaddam MR. A Systematic Review for Estimation of HTLV-I Infection in the Blood Donors of Iran. Iran J Basic Med Sci. 2013;16(3):196-201. [PubMed: 24470861].

9. Sadeghi F, Bokharaei-Salim F, Salehi-Vaziri M, Monavari SH, Alavian SM, Salimi S, et al. Associations between human TRIM22 gene expression and the response to combination therapy with Peg-IFNalpha-2a and ribavirin in Iranian patients with chronic hepatitis C. J Med Virol. 2014;86(9):1499-506. doi: 10.1002/jmv.23985. [PubMed: 24889558].

10. Salehi-Vaziri M, Sadeghi F, Alamsi-Hashiani A, Haeri H, Monavari $\mathrm{SH}$, Keyvani H. Merkel cell polyomavirus and human papillomavirus infections in cervical disease in Iranian women. Arch Virol. 2015;160(5):1181-7. doi: 10.1007/s00705-015-2368-4. [PubMed: 25721299].

11. Naderi M, Paryan M, Azadmanesh K, Rafatpanah H, Rezvan H, Mirab Samiee S. Design and development of a quantitative real time PCR assay for monitoring of HTLV-1 provirus in whole blood. J Clin Virol. 2012;53(4):302-7. doi: 10.1016/j.jcv.2011.12.033. [PubMed: 22306271].

12. Hedayati-Moghaddam MR, Fathimoghadam F, Eftekharzadeh Mashhadi I, Soghandi L, Bidkhori HR. Epidemiology of HTLV-1 in Neyshabour, Northeast of Iran. Iran Red Crescent Med J. 2011;13(6):4247. [PubMed: 22737506].

13. Azarpazhooh MR, Hasanpour K, Ghanbari M, Rezaee SA, Mashkani B, Hedayati-Moghaddam MR, et al. Human T-lymphotropic virus type 1 prevalence in northeastern Iran, Sabzevar: an epidemiologicbased study and phylogenetic analysis. AIDS Res Hum Retroviruses. 2012;28(9):1095-101. doi:10.1089/AID.2011.0248. [PubMed: 22229796].

14. Khameneh ZR, Baradaran M, Sepehrvand N. Survey of the seroprovalence of HTLVI/II in hemodialysis patients and blood donors in Urmia. Saudi J Kidney Dis Transpl. 2008;19(5):838-41. [PubMed: 18711311].

15. Rafatpanah H, Torkamani M, Valizadeh N, Vakili R, Meshkani B, Khademi H, et al. Prevalence and phylogenetic analysis of HTLV-1 in a segregated population in Iran. J Med Virol. 2016;88(7):1247-53. doi: 10.1002/jmv.24448. [PubMed: 26680556].

16. Ajami A, Hosseini RF, Tabarestani N. Seroepidemiological survey of HTLV-I/II in blood donors of Mazandaran in 1999 [In Persian].J Mazandaran Univ Med Sci. 2000;10(26):45-8.

17. Ghaffari J, Kowsarian M, Mahdavi M, Vahid Shahi K, Rafatpanah H, Tafreshian A. Prevalence of HTLV-I infection in patients with thalassemia major in Mazandaran, North of Iran.Jundishapur J Microbiol. 2012;6(1):57-60. doi: 10.5812/jjm.4702. 